\title{
Survival rate prediction of breast cancer patients of 0-IV stages with and without radiotherapy via a revised Taylor series expansion algorithm: A population-based study in Taiwan
}

\author{
Shao-Wen Chiu ${ }^{\mathrm{a}, \mathrm{b}}$, Jia-Feng Peng ${ }^{\mathrm{c}}$, Tzu-Hwei Wang ${ }^{\mathrm{d}, \mathrm{e}}$, Lung-Fa Pan ${ }^{\mathrm{a}, \mathrm{f}, *}$ and \\ Lung-Kwang Pan ${ }^{\text {a,* }}$ \\ ${ }^{a}$ Graduate Institute of Radiological Science, Central Taiwan University of Science and Technology, \\ Takun, Taichung 406, Taiwan \\ ${ }^{\mathrm{b}}$ Medical Care Department, Healthcare Technology Business Division, International Integrated \\ Systems, Inc., New Taipei 345, Taiwan \\ ${ }^{\mathrm{c}}$ Animal Quarantine Division, Bureau of Animal and Plant Health Inspection and Quarantine, Council \\ of Agriculture, Executive Yuan, Taipei 100, Taiwan \\ ${ }^{\mathrm{d}}$ Department of Radiation Oncology, Yee Zen Hospital, Taoyuan 326, Taiwan \\ ${ }^{\mathrm{e}}$ Department of Radiation Oncology, Buddhist Tzu Chi General Hospital, Hualien 970, Taiwan \\ ${ }^{\mathrm{f}}$ Department of Cardiology, Taichung Armed Forces General Hospital, Taichung 402, Taiwan
}

\begin{abstract}
.
BACKGROUND: The morbidity of breast cancer has continuously achieved a global topicality. In particular, during the last decade several ten thousand female adults in Taiwan have been confirmed as breast cancer patients.

OBJECTIVE: To predict the survival rate of breast cancer patients at various (0-IV) stages and provide efficient assessment of proposed radiotherapy for patients.

METHODS: The prediction algorithm proposed is based on the revised hit and target model and implies the application of Taylor series expansion to the population-based survey dataset. The proposed algorithm features a specific function comprising a single simple exponential term $\exp (-\alpha t)$ to imply the fundamental degradation of patient's health multiplied by an additional term $P(\alpha t)$, which specifies the recovery effect of a particular therapy.

RESULTS: Its calculated values for breast cancer patients who undergone radiotherapy at different stages 0-IV were $\{0.0029$, $0.0066,0.0178,0.0475,0.1785 \mathrm{yr}^{-1}$, respectively, while those for corresponding groups of patients with no radiotherapy were assessed as $\{0.0072,0.0137,0.0264,0.0913,0.2425\} \mathrm{yr}^{-1}$.

CONCLUSIONS: The revised algorithm successfully interpreted the breast cancer patients' survival rate at stages 0-IV and evaluated the necessity of radiotherapy for patients at various stages as well.
\end{abstract}

Keywords: Survival rate, breast cancer, algorithm, Taylor series expansion, hit and target model

\footnotetext{
${ }^{*}$ Corresponding authors: Lung-Fa Pan and Lung-Kwang Pan, Graduate Institute of Radiological Science, Central Taiwan University of Science and Technology, Takun, Taichung 406, Taiwan. E-mails: lung-fa@803.org.tw, 1kpan@ @ctust.edu.tw.
}

0928-7329/19/\$35.00 (C) 2019 - IOS Press and the authors. All rights reserved

This article is published online with Open Access and distributed under the terms of the Creative Commons Attribution NonCommercial License (CC BY-NC 4.0). 


\section{Introduction}

The morbidity of breast cancer has continuously achieved a global topicality. In particular, during the last decade several ten thousand female adults in Taiwan have been confirmed as breast cancer patients, yet, even the state-of-the-art medical care fails to mitigate the mortality rate of nearly 2,000 persons per year [1]; so high lethal rate of the breast cancer is also related to its aggravation of chronic diseases like diabetes [2]. The early diagnostics of breast cancer is given the top priority status in Taiwan, which Department of Health (DOH) provides free annual mammographic screening for females over 40 years (X-ray or ultrasonic one for the age range from 40 to 50, X-ray only for female patients over 50) [3]. Many researchers and public health officers addressed the issue of early detection of breast cancer using a limited budget, in order to drop the mortality rate.

The comprehensive analysis of bountiful information obtained from many perspective surveys is also instrumental in guiding the public health strategies on a wide assistance to breast cancer patients. Liu et al. adopted the joint point regression analysis to trace the annual relative variations of incidence, prevalence, and survival outcome for breast cancer patients [4]. Huang and Yen substantiated the notion that a medium level of radiotherapy was usually helpful for breast cancer patients who underwent surgical operation, since it was reported to effectively improve the survival rate [5]. Chuang et al. investigated the survival rate for breast cancer patients at $N_{1}$ stage from the cancer registry data bank and revealed a higher survival rate of patients undergone radiotherapy treatment, as compared to those who did not [6]. Wu et al. analyzed the data of breast cancer patients from 1973 to 2012 and reported that radiotherapy improved both cause-specific survival (CSS) and overall survival (OS) of patients that underwent the respective surgical operation [7].

Thus, the primary goal of this study was to predict the effect of different factors, including therapies, on the survival rate of breast cancer patients based on the huge data array obtained from Taiwan population survey of 2002-2017. The secondary goal was to validate the feasibility of the proposed approach using results of other scholars on other (rectum and colon) cancer cases and formulate solid recommendations based on the comprehensive interpretation of the quantified results. In doing so, the survival rate versus year was predicted, according to the Taylor series expansion algorithm, while the lethal frequency $\left[\mathrm{yr}^{-1}\right]$ was introduced to help quantify the predicted and actual results. The revised algorithm successfully interpreted the breast cancer patients' survival rate at stages 0-IV and evaluated the necessity of radiotherapy for patients at various stages as well.

\section{Material and methods}

\subsection{Revised survival function via the Taylor series expansion algorithm}

The survival rate of breast cancer patients can be derived via the Taylor series expansion. The algorithm is revised from the conventional hit and target model that is widely used for deriving the survival probability of a cell with direct and indirect action of different factors controlling DNA damage (see Eq. (1)). Accordingly, the survival rate $P(n)$ is assumed to be the sum of many cases with various probabilities and can be implied as a Taylor series expansion (see Eq. (2)). Although the first term $\exp (-\alpha t)$ of these series is always dominant in this algorithm, the following terms also provide minor contributions with lower and lower probabilities as defined in Eqs (1) and (2). Thus, the revised survival rate can be defined via Eqs (3)-(6) to imply the probability variations in the actual data analysis. 


$$
\begin{aligned}
& \sum_{n=0}^{\infty} P(n) \equiv 1=e^{-\alpha \cdot t} \cdot e^{\alpha t}=e^{-\alpha t} \cdot P(\alpha t) \\
& \quad=e^{-\alpha t} \cdot\left(1+\alpha t+\frac{1}{2 !}(\alpha t)^{2}+\frac{1}{3 !}(\alpha t)^{3}+\frac{1}{4 !}(\alpha t)^{4}+\ldots\right)
\end{aligned}
$$

Thus, the survival rate is derived as follows:

$$
=e^{-\alpha_{0} t}=e^{-\frac{1}{t} t}=e^{-1}=0.37
$$

(patient expired because of disease progression)

$=e^{-\alpha_{1} t} \cdot\left(1+\alpha_{1} t\right)=0.37$

(patient had satisfactory response to therapy)

$$
=e^{-\alpha_{2} t} \cdot\left(1+\alpha_{2} t+\frac{1}{2 !}\left(\alpha_{2} t\right)^{2}\right)=0.37
$$

(patient had good response to therapy)

$$
\begin{aligned}
& =e^{-\alpha_{3} t} \cdot\left(1+\alpha_{3} t+\frac{1}{2 !}\left(\alpha_{3} t\right)^{2}+\frac{1}{3 !}\left(\alpha_{3} t\right)^{3}\right)=0.37 \\
& \text { (patient had excellent response to therapy) }
\end{aligned}
$$

Define term $\alpha_{i}$ in Eqs (3)-(6) as the lethal frequency $\left[\mathrm{yr}^{-1}\right]$, which is the reciprocal $(1 / t)$ of the specific time $t$ in Eq. (3). The specific time is derived for the survival rate reaching 0.37 in reality. This is defined as Scenario I, which implies that "patient expired because of disease progression" since no apparent treatment improved the survival rate and, thus, the patient's death pattern followed a simple exponential term. In Scenario II, which implies that "patient had satisfactory response to therapy", the lethal frequency $\alpha_{1}$ is not reduced to $1 / t$ but involves the whole expression as denoted in Eq. (4) to reach 0.37 , whereas the specific time $t$ still can be derived from the real survival rate reaching 0.37 . Scenario II is sustained if the patient's survival rate is improved by therapy, and a slight shoulder effect can be obtained from the former survival rate. Further, if the specific time $t$ is fixed in Scenarios I or II, a simple computation (e.g., via Excel) yields that term $\alpha_{1}$ exceeds $\alpha_{0}$ by 2.14 times. Lethal frequencies $\alpha_{2}$ and $\alpha_{3}$ can be similarly derived for Scenario III ("patient had good response to therapy") and Scenario IV ("patient had excellent response to therapy"): the calculated values $\alpha_{2}$ and $\alpha_{3}$ exceed $\alpha_{0}$ by 3.25 and 4.34 times, respectively. Noteworthy is that all lethal frequencies are derived for the specific time $t$ and the same survival rate of 0.37 as indicated in Eqs (3)-(6). Once the specific time is fixed, each respective term $\alpha_{i}$ can be accurately calculated.

Figure 1 shows four kinds of prediction to imply different scenarios of survival rate corresponding to various levels of recovery. As clearly shown in Fig. 1, "normal exponential term" implies no additional recovery and zero shoulder effect. "Satisfactory response to therapy" indicates a satisfactory recovery of a patient, while a slight shoulder effect implies the improvement of additional survival rate. Either "good response" or "excellent response" implies good or excellent recovery of a patient, while and a medium or strong shoulder effect supports the increasing survival rate in reality.

A similar assumption was used in the hit and target model [8], which successfully interpreted the survival probability of a cell from the microscopic viewpoint. Restated, the revised algorithm is supposed to improve the prediction of survival rate from a massive data group and to interpret it from the statistical viewpoint on the basis of the Taylor series expansion. It makes use of a similarity between life predictions 
S112 S.-W. Chiu et al. / Survival rate prediction of breast cancer patients of 0-IV stages with and without radiotherapy

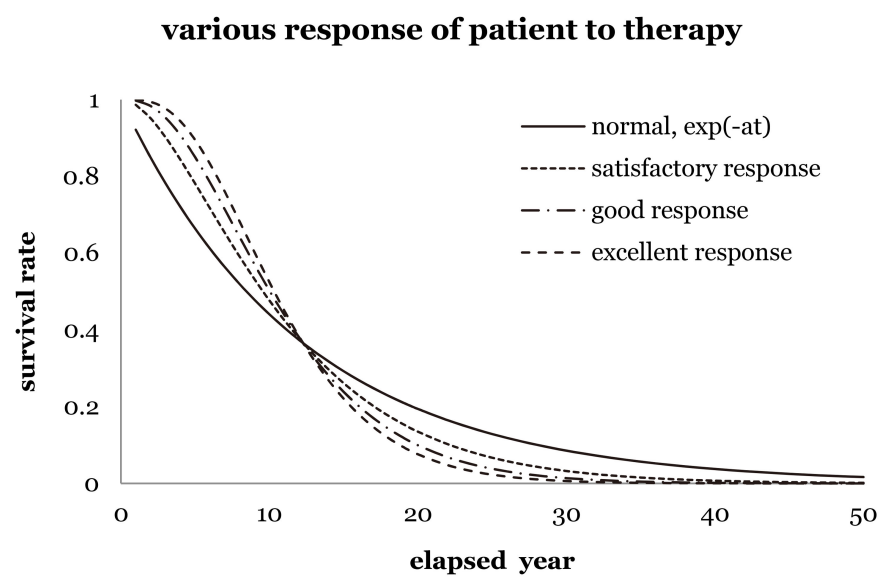

Fig. 1. The survival rate of breast cancer female patients versus elapsed years for different responses to therapy (satisfactory, good, and excellent). For instance, set the lethal frequency is the inverse of the elapsed time of $12.20 \mathrm{yr}$ of the normal exponential function, $\exp (-\alpha t)$, e.g., if the survival rate reaches 0.37 after $12.20 \mathrm{yr}$, the lethal frequency is 0.082 . However, various shoulder effects appear if the patients have satisfactory, good or excellent response to the radiotherapy progression, although each curve still reaches 0.37 after $12.20 \mathrm{yr}$.

of cancer patients and vital radionuclides with an average decay constant $\lambda\left[t^{-1}\right]$. Within framework of this similarity approach, the survival rate of a cancer patient, $e^{-\alpha t}$, has the same pattern as the activity of radionuclide, $A(t)=A_{0} \cdot e^{-\lambda t}$; where the lethal case (patient's death) corresponds to the radionuclide decay from its active status. A large decay constant $(\lambda)$ of radionuclide implies a short decay half-life $\left(T_{1 / 2}=\ln 2 / \lambda\right)$ and causes a rapid decay in a short period of time [9]; whereas a large lethal frequency $\left(\alpha\left[t^{-1}\right]\right)$ implies a low survival rate of cancer patient's progression in a short period of time.

\subsection{Data sorting for breast cancer patients}

Figure 2 shows the flow chart of the data sorting in this study. The analyzed data were collected and sorted mainly from the Department of Health (DOH), Taiwan nationwide cancer registry data bank with de-identification process to create the broken point for personnel tracking. The total data for breast cancer patients were collected from 2002 to 2017, yet the analysis covered only the data starting from 2007 due to their completeness in the entire essential data recording format. The formal registered data for breast cancer patients (i.e., registry data following the code number C50.0-C50.9 by ICD-O-3 criteria) contained 141,019 events from 2007 to 2015 [10], which were subjected to the survival rate analysis at various stages in this study. Accordingly, one event was defined as one specific case dead and being recorded in the cancer registry data bank as an available "sensor" data. If the objects (cases) were still alive on December 31, 2015, then they were recorded as active and vital. The collected data were specifically focused on the cases diagnosed as breast cancer C50.0-C50.9 and excluded outliers [11], which fell into any of the following categories: (1) those excluded by hospital (207 cases), (2) not females (373 cases), (3) records were beyond the range of $2007 \sim 2015$ (1747 cases), (4) not class 1 nor class 2 according to code C50.0-C50.9 by ICD-O-3 criteria (30767 cases), (5) age beyond 15-120 (3 cases), (6) non-histology type $\mathrm{M}$ (827 cases), (7) failure to calculate the survival time (36 cases), (8) duplicate cases (12545 cases), and (9) failure to classify cancer stage (273 cases). Thus, the total number of 94,243 events was successfully recorded and subjected to the further analysis (cf. Fig. 2). The events were later categorized by patients who underwent radiotherapy (WRT) or not (NRT), and were subjected or not to 
S.-W. Chiu et al. / Survival rate prediction of breast cancer patients of 0-IV stages with and without radiotherapy S113

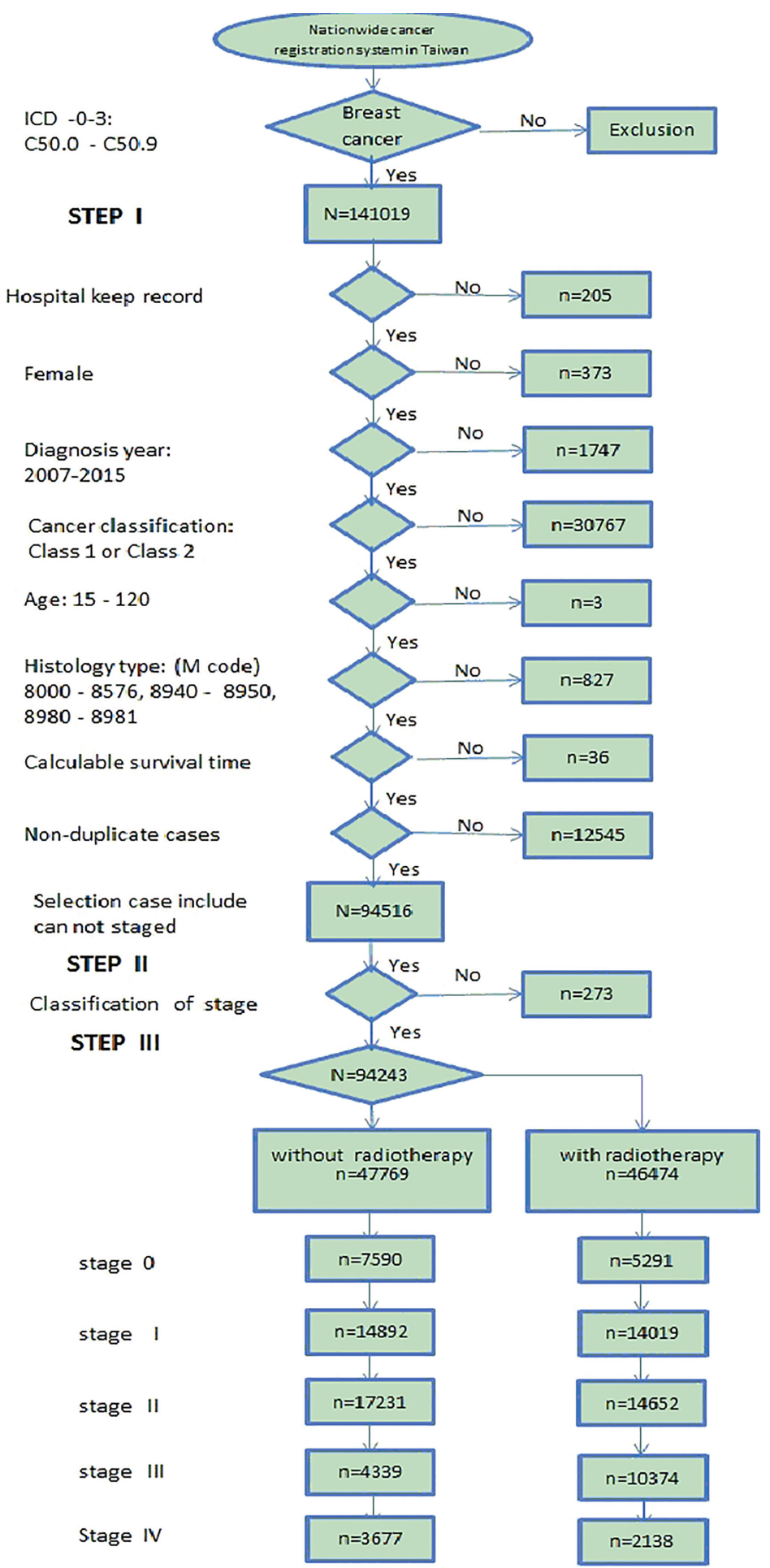

Fig. 2. The flowchart of the data sorting in this study. The data were collected from 2007 to 2015. 
S114 S.-W. Chiu et al. / Survival rate prediction of breast cancer patients of 0-IV stages with and without radiotherapy

Table 1

The breakdown of breast cancer female patients by various stages (0-IV) in Taiwan, according to data collected from 2007 to 2015

\begin{tabular}{lccc}
\hline Stage & Without radiotherapy & With radiotherapy & Subtotal \\
\hline 0 & 7600 & 5291 & 12891 \\
I & 14892 & 14019 & 28911 \\
II & 17241 & 14652 & 31893 \\
III & 4349 & 10374 & 14723 \\
IV & 3687 & 2138 & 5825 \\
Total & 47769 & 46474 & 94243 \\
\hline
\end{tabular}

Table 2

Classification of various stages of breast cancer, in accordance with [11], where terms $\mathrm{T}, \mathrm{N}$ and $\mathrm{M}$ denote tissue, lymph, and metastases, respectively

\begin{tabular}{|c|c|}
\hline Stage & General definition \\
\hline \multirow[t]{3}{*}{0} & $\mathrm{~T}_{\mathrm{is}}$ : carcinoma in situ. \\
\hline & $\mathrm{N}_{0}$ : No regional lymph node metastasis. \\
\hline & $\mathrm{M}_{0}$ : No clinical or radiographic evidence of distant metastases. \\
\hline \multirow[t]{3}{*}{ I } & $\mathrm{T}_{1}$ : Tumor $\leqslant 20 \mathrm{~mm}$ or less in greatest dimension. \\
\hline & $\mathrm{N}_{0}$ : No regional lymph node metastasis. \\
\hline & $\mathrm{M}_{0}$ : No clinical or radiographic evidence of distant metastases. \\
\hline \multirow[t]{2}{*}{ II } & $\mathrm{T}_{2,3}: 20 \mathrm{~mm} \leqslant$ tumor $\leqslant 50 \mathrm{~mm}$; or tumor $>50 \mathrm{~mm}$ in greatest dimension. \\
\hline & $\begin{array}{l}\mathrm{N}_{0,1} \text { : No regional lymph node metastasis; or Metastases to movable ipsilateral level I, II axillary lymph node(s). } \\
\mathrm{M}_{0} \text { : No clinical or radiographic evidence of distant metastases. }\end{array}$ \\
\hline \multirow[t]{3}{*}{ III } & $\begin{array}{l}\mathrm{T}_{1 \sim 4} \text { : Tumor } \leqslant 20 \mathrm{~mm} \text { or less in greatest dimension; or } 20 \mathrm{~mm} \leqslant \text { Tumor } \leqslant 50 \mathrm{~mm} \text {; or Tumor }>50 \mathrm{~mm} \text { in } \\
\text { greatest dimension; or Tumor of any size with direct extension to the chest wall and/or to the skin (ulceration or } \\
\text { skin nodules). }\end{array}$ \\
\hline & $\begin{array}{l}\mathrm{N}_{1,2} \text { : Metastases to movable ipsilateral level I, II axillary lymph node(s); or metastases in ipsilateral level I, II } \\
\text { axillary lymph nodes that are clinically fixed or matted; or in clinically detected ipsilateral internal mammary } \\
\text { nodes in the absence of clinically evident axillary lymph node metastases. }\end{array}$ \\
\hline & $\mathrm{M}_{0}$ : No clinical or radiographic evidence of distant metastases. \\
\hline \multirow[t]{5}{*}{ IV } & $\begin{array}{l}\mathrm{T}_{3,4}: \text { Tumor }>50 \mathrm{~mm} \text { in greatest dimension; or tumor of any size with direct extension to the chest wall and/or to } \\
\text { the skin (ulceration or skin nodules). }\end{array}$ \\
\hline & $\mathrm{N}_{3}$ : Metastases in ipsilateral infraclavicular (level III axillary) lymph node(s) with or without level I, II axillary \\
\hline & lymph node involvement; or in clinically detected ipsilateral internal mammary lymph node(s) with clinically \\
\hline & $\begin{array}{l}\text { evident level I, II axillary lymph node metastases; or metastases in ipsilateral supraclavicular lymph node(s) with } \\
\text { or without axillary or internal mammary lymph node involvement. }\end{array}$ \\
\hline & $\begin{array}{l}\mathrm{M}_{1} \text { : Distant detectable metastases as determined by classic clinical and radiographic means and/or histologically } \\
\text { proven larger than } 0.2 \mathrm{~mm} \text {. }\end{array}$ \\
\hline
\end{tabular}

surgical operation or other treatments at various cancer stages. Accordingly, the breakdown of events for breast cancer patients at stages 0-IV into two classes (WRT and NRT) is listed in Table 1 (cf. Fig. 2). Furthermore, the major definition of stages 0-IV of breast cancer patients from [11] is listed in Table 2, whereas the respective treatments for these stages are given in Table 3. Most treatments were recommended by $\mathrm{DOH}$, since each protocol had to be verified by the high professional medical experts in the latter process, in order with comply with the public health policy and budget control in Taiwan.

The most adopted definition of survival rate is defined as overall survival (OS), which is also referred to as observed survival. Accordingly, the purpose was to accurately predict the residual time for one event (e.g., death of a breast cancer female patient, irrelevant of its cause). This was also the most acknowledged model to assess the expediency of the respective treatment for prolonging the patient's life from the clinical viewpoint. In this study, the analytical calculation was performed using Statistical Analysis System (SAS V9.4) software developed by SAS Institute (US) [12], while the computation of survival rate was performed via the Kaplan-Meier method, which description can be found elsewhere [13]. 
Table 3

Major treatments for patients with breast cancer in Taiwan

\begin{tabular}{|c|c|c|}
\hline Stage & $\begin{array}{l}\text { Major treatment for patients } \\
\text { with no radiotherapy (NRT) }\end{array}$ & $\begin{array}{l}\text { Major treatment for patients } \\
\text { with radiotherapy (WRT) }\end{array}$ \\
\hline \multirow[t]{2}{*}{0} & MRM (modified radical mastectomy) & BCS (breast conservative treatment) + RT (radiotherapy) \\
\hline & Hormone therapy if necessary & Hormone therapy if necessary \\
\hline \multirow[t]{3}{*}{ I } & MRM + ALND (axillary lymph node dissection) or & $\mathrm{BCS}+\mathrm{RT}$ \\
\hline & SLND (sentinel lymph node dissection) & Adjuvant treatment by pathologic result $(\mathrm{e}$ \\
\hline & $\begin{array}{l}\text { Adjuvant treatment by pathologic result (ex: } \\
\text { chemotherapy, hormone therapy, and target therapy) }\end{array}$ & chemotherapy, hormone therapy, and target therapy) \\
\hline \multirow[t]{2}{*}{ II } & MRM + ALND or SLND & $\mathrm{BCS}+\mathrm{RT}$ \\
\hline & $\begin{array}{l}\text { Adjuvant treatment by pathologic result (ex: } \\
\text { chemotherapy, hormone therapy, and target therapy) }\end{array}$ & $\begin{array}{l}\text { Adjuvant treatment by pathologic result (ex: } \\
\text { chemotherapy, hormone therapy, and target therapy) }\end{array}$ \\
\hline III & Similar treatment as for stage II & $\begin{array}{l}\text { Neoadjuvant chemotherapy if large tumor size then BCS } \\
\text { or MRM + ALND or SLND + RT } \\
\text { Adjuvant treatment by pathologic result (ex: } \\
\text { chemotherapy, hormone therapy, and target therapy) }\end{array}$ \\
\hline IV & $\begin{array}{l}\text { Systemic treatment (ex: chemotherapy, hormone } \\
\text { therapy and target therapy) }\end{array}$ & $\begin{array}{l}\text { Systemic treatment (ex: chemotherapy, hormone therapy, } \\
\text { and target therapy) + RT }\end{array}$ \\
\hline
\end{tabular}

\section{Results}

\subsection{Survival rate of stages 0-IV of breast cancer patients}

Figure 3 illustrates various survival rates of breast cancer patients of stages 0-IV as recorded from 2007 to 2015. The exponential trend of the survival rate can be easily regressed by Excel default feature for Major treatment for patients with radiotherapy (WRT) and Major treatment for patients with no radiotherapy (NRT) patients at stages 0-IV and had a high coefficient of determination, $r^{2}(0.97-0.99)$ as listed in Table $4[14,15]$. In addition, the power $\left(\alpha_{i}\right)$ of the exponential function was defined as lethal frequency, while the reciprocal of this frequency was the average life of the patient at the particular stage of breast cancer (cf. Eq. (3)). As seen in Table 3, the average life degraded gradually for breast cancer patients from stage 0 to IV and WRT patients always had a higher average life than NRT ones. A comparative analysis of predicted average life values of WRT and NRT patients at stages 0 or I revealed no significant differences, since both exceeded 70 years (cf. Table 3, stage 0: 139.7 vs. $340.1 \mathrm{yr}$; stage I: 72.9 vs. $150.6 \mathrm{yr}$ for WRT and NRT patients, respectively). Much larger differences in the average life values were observed for patients at stages II ( $37.8 \mathrm{vs}$. $56.3 \mathrm{yr})$ and III (11.0 vs. $21.0 \mathrm{yr})$ but were reduced at stage IV ( $4.1 \mathrm{vs.} 5.6 \mathrm{yr}$ ). Thus, patients at cancer stages II or III were strongly recommended to undergo radiotherapy, in order to improve their survival rate. In contrast, the radiotherapy application to patients at stage IV could ensure only a slight prolongation of residual life (by 1.5 years).

\subsection{Taylor series expansion algorithm for patients at stage IV}

To further inspect the predicted survival rate at stage IV in Fig. 3E, two thin lines (solid and doted ones) represent the linear regressed lines of raw data from Excel default feature for WRT and NRT patients, respectively, whereas another two thick lines (dashed-and-dotted and dotted ones) implied the theoretical predictions, according to the revised algorithm that assumed a satisfactory response to treatment for WRT and NRT patients, respectively (cf. Eq. (4)). As seen in Fig. 3 (E, stage IV), thick and thin lines exhibited a medium difference in the preliminary section. The derived shoulder effect disagreed with the collected events in either two groups, since a simple exponential term possessed a high 
S116 S.-W. Chiu et al. / Survival rate prediction of breast cancer patients of 0-IV stages with and without radiotherapy

(A) stage 0

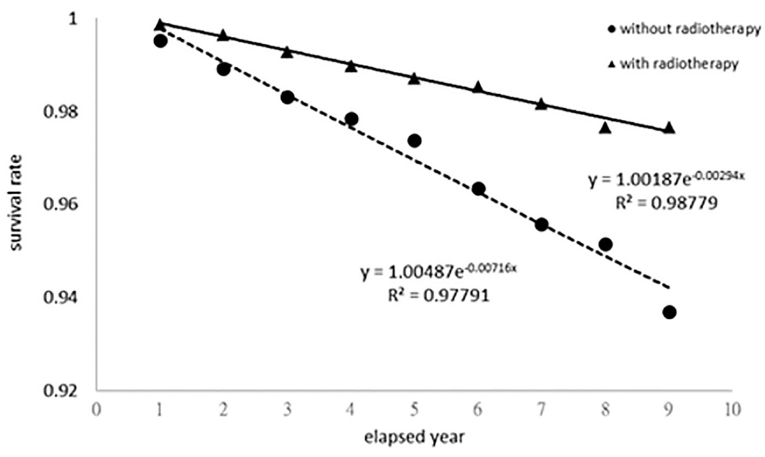

(C) stage II

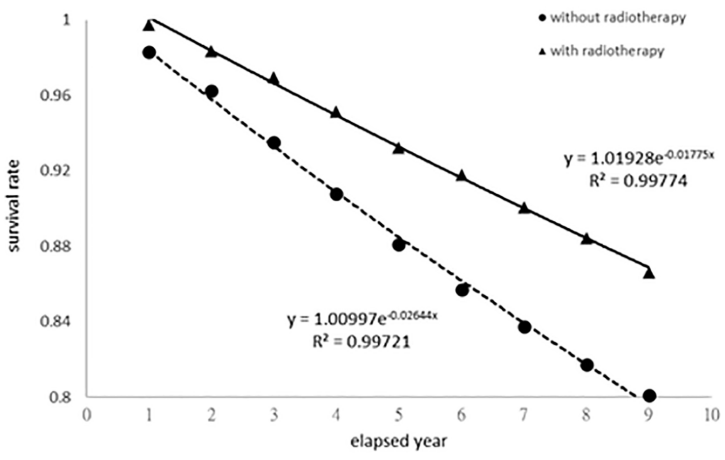

(B) stage I

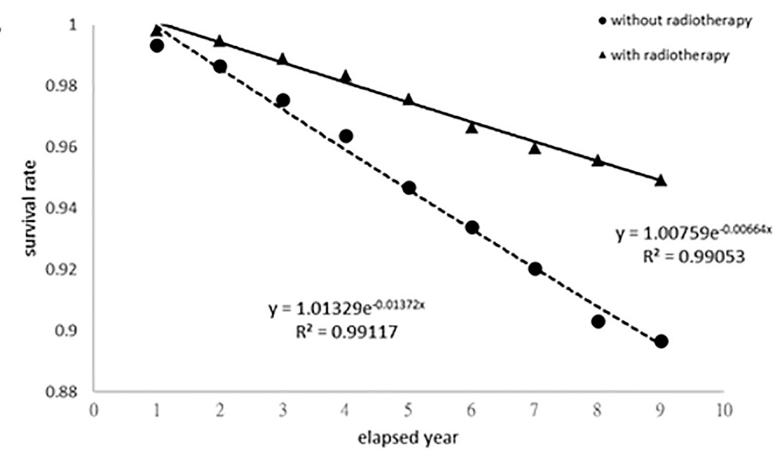

(D) stage III

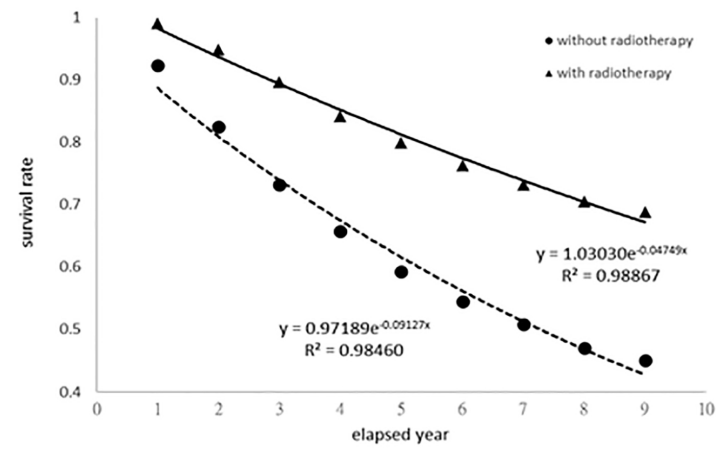

(E) stage IV

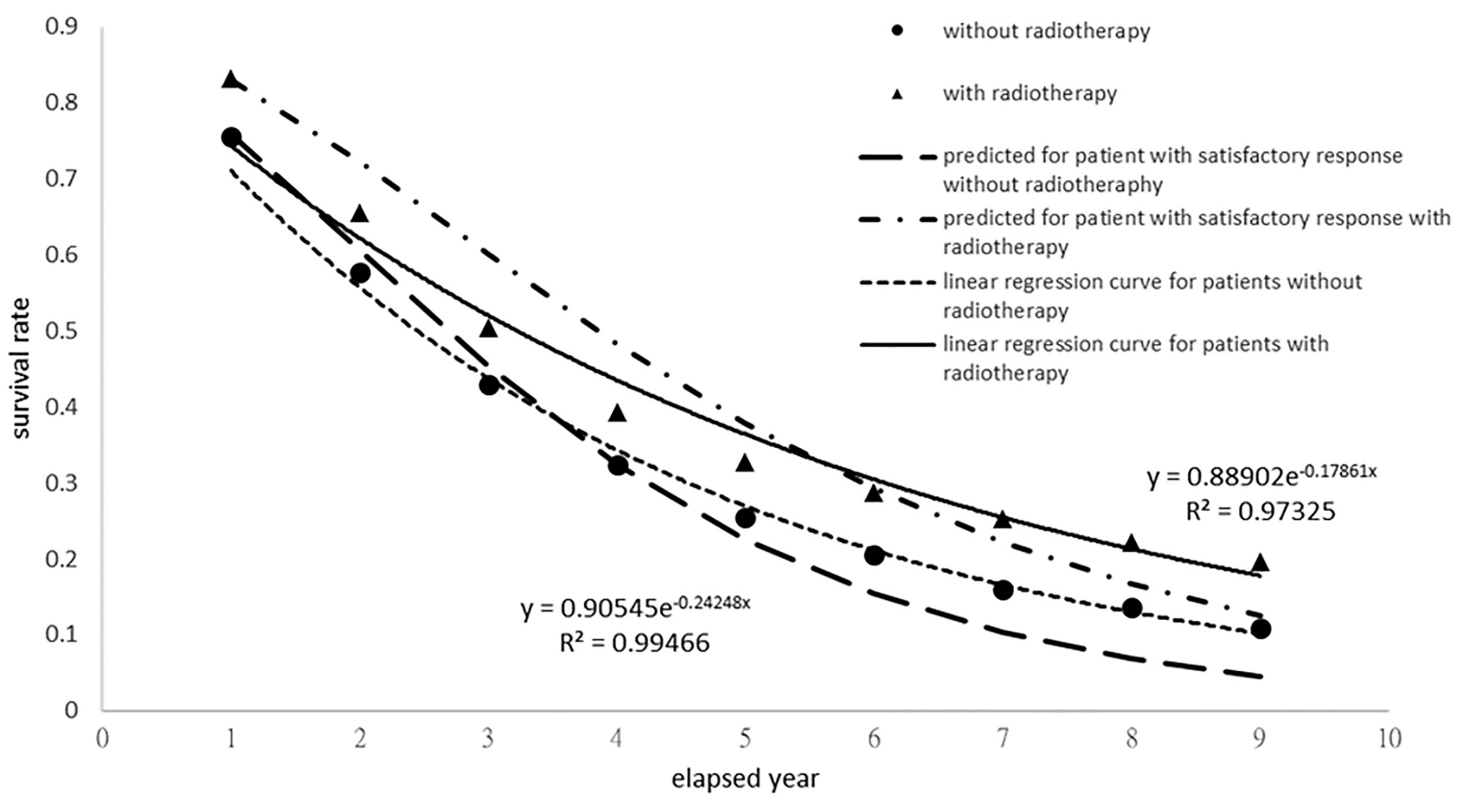

Fig. 3. The survival rate of breast cancer patients in Taiwan with breakdown by various (0-IV) stages. The revised algorithm accurately predicted the survival rate with the lethal frequency estimation. 
S.-W. Chiu et al. / Survival rate prediction of breast cancer patients of O-IV stages with and without radiotherapy S117

Table 4

Lethal frequency and average life estimations for breast cancer patients in Taiwan with breakdown by 0-IV stages

\begin{tabular}{|c|c|c|c|c|c|c|}
\hline \multirow[t]{2}{*}{ Stage } & \multicolumn{2}{|c|}{ Coefficient of determination, $r^{2}$} & \multicolumn{2}{|c|}{ Lethal frequency, $\alpha\left[\mathrm{yr}^{-1}\right]$} & \multicolumn{2}{|c|}{ Average life, $1 / \alpha$ [yr] } \\
\hline & No radiotherapy (NRT) & With radiotherapy (WRT) & NRT & WRT & NRT & WRT \\
\hline 0 & 0.97791 & 0.98779 & 0.0072 & 0.0029 & 139.7 & 340.1 \\
\hline I & 0.99117 & 0.99053 & 0.0137 & 0.0066 & 72.9 & 150.6 \\
\hline II & 0.99721 & 0.99774 & 0.0264 & 0.0178 & 37.8 & 56.3 \\
\hline III & 0.98460 & 0.98867 & 0.0913 & 0.0475 & 11.0 & 21.0 \\
\hline IV & 0.99466 & 0.97325 & 0.2425 & 0.1786 & 4.1 & 5.6 \\
\hline $\mathrm{IV}^{*}$ & & & 0.5190 & 0.3822 & 1.9 & 2.6 \\
\hline
\end{tabular}

${ }^{*}$ The revised algorithm implied that patients with or without radiotherapy had satisfactory responses.

coefficient of determination $r^{2}$ ( 0.9947 for WRT patients and 0.9733 for NRT ones). Thus, the revised algorithm did not provide more accurate prediction in evaluating breast cancer patients at stage IV, or from another viewpoint, the radiotherapy for patients at stage IV did not provide significant contribution to the improvement of patients' health and residual life.

\section{Discussion}

\subsection{Regrouping breast cancer patients at stage IV sorted by surgical operations}

The events of breast cancer patient at stage IV can be regrouped and sorted by surgical operations. Accordingly, the new group of follow-up events at various stages undergone surgical operation or not versus annual year is $\{2376,2143,1867,1627,1481,1411,1371,1343,1334\}$ and $\{3439,2445,1918,1598$, $1421,1334,1296,1278,1271\}$. Nevertheless, the total number of events is still $5815(5818=2376+$ $3439)$ and equals to that of the original two groups sorted by radiotherapy $(5815=2138+3677)$. Figure 4 shows the new survival rate versus year for patients that underwent surgical operation or not. The lethal frequency becomes 0.2750 and $0.1572 \mathrm{yr}^{-1}$ (cf. Table 3, stage IV, original $0.2425,0.1786 \mathrm{yr}^{-1}$ ). In addition, the average life also changes to 3.6 and $6.4 \mathrm{yr}$, respectively, as compared to original 4.1 and 5.6 yr. As seen in Fig. 4, the derived shoulder effect did not manifest itself for the re-grouped data of patients that underwent surgical operation or not, since both coefficients of determination reached 0.97 . In other words, neither radiotherapy nor surgical operation provides medical treatment with satisfactory response for breast cancer patients at stage IV. However, the surgical operation is still recommended since one should expect that it will prolong the patient's average life by nearly $78 \%$ (from 3.6 to $6.4 \mathrm{yr}$ ), while radiotherapy can extend it only by $37 \%$ (from 4.1 to $5.6 \mathrm{yr}$ ). Thus, either radiotherapy or surgical operation for breast cancer patients at stage IV can temporarily suppress the clinical syndrome, but the respective patients are most likely to expire (cf. Eq. (3)), due to their unsatisfactory response to the medical treatment (cf. Eq. (4)).

\subsection{Interpretation of the survival rate of colon and rectum cancer patients at stage IV}

The survival rate for colon and rectum cancer patients at stage IV registered in Taiwan in the last decade was also analyzed and plotted in Fig. 5, according to the same algorithm. The data were collected from 2007 to 2015 with the screening code C18-C20 in the same ICD-O-3 data bank. The colon and rectum cancer patients were sorted into two classes: those undergone surgical operation or not; then the collected data versus annual period correspond to $\{13672,10011,7110,5570,4930,4629,5630,4494$, 


\section{breast cancer stage IV breakdown by patients that underwent surgical operation or not}

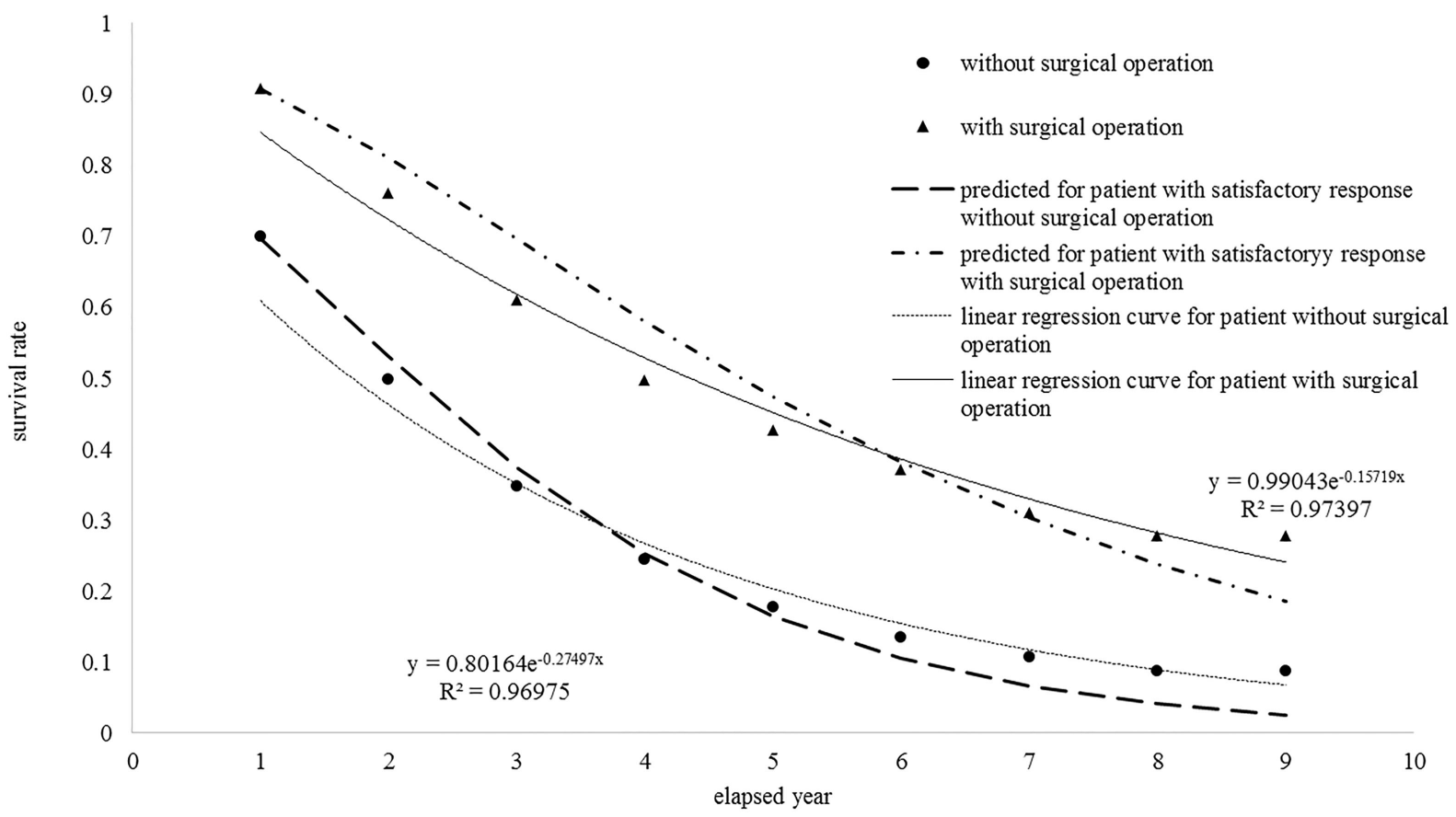

Fig. 4. The same group of breast cancer patients at stage IV was reorganized with breakdown by those who underwent surgical operation or not and then re-plotted versus the elapsed year.

$4478\}$ and $\{7725,2999,1521,1148,1052,1017,1003,997,995\}$. The total number taken into account was $21487(21397=13672+7725)$ for Stage IV patients, whereas the total amount of colon and rectum cancer patients at all stages registered in Taiwan during the nine-year period from 2007 to 2015 was 104,380 persons with an average of about 11,600 patients per year. The respective survival rate versus year is depicted in Fig. 5. The default linear regression feature provided by Excel software suggests a simple exponential term with a medium coefficient of determination $r^{2}(0.903$ or 0.929 for patients undergone surgical operation or not, respectively), whereas the revised algorithm also provided a good prediction of both survival rates versus year. The shoulder effect provided by this algorithm perfectly fits the annual clinical data. The lethal frequency also changed from original 0.545 and $0.258 \mathrm{yr}^{-1}$ denoted as "patient expired because of disease progression" to 1.166 and $0.552 \mathrm{yr}^{-1}$ "patient had satisfactory response to therapy" (cf. Eqs (3) and (4)). In addition, the respective average life of patient changed from 1.8 and $3.9 \mathrm{yr}$ to 0.9 and $1.8 \mathrm{yr}$ to better interpret the actual collected data for colon and rectum cancer patients at stage IV who underwent surgical operation or not. The accurate prediction of survival rate can effectively provide $\mathrm{DOH}$ or other related research institutions to optimize the budget in an efficient way.

\subsection{Re-evaluation of survival rate data from other sources}

The same algorithm was also tested on survival rate data of other researchers' listed in Table 5. Noteworthy is that the overall survival rate can be easily replaced by quantified lethal frequency or average life to represent its unique characteristics. In addition, none of these performances shows a satisfactory 
S.-W. Chiu et al. / Survival rate prediction of breast cancer patients of 0-IV stages with and without radiotherapy S119

Table 5

Application of the proposed revised algorithm to data from [7,16,17] with estimation of lethal frequency, average life, and respective cancer progress scenario

\begin{tabular}{|c|c|c|c|c|c|c|}
\hline Source & Study subject & Definition & Specific description & Scenario & $\begin{array}{l}\text { Lethal } \\
\text { frequency } \\
{\left[\mathrm{yr}^{-1}\right]}\end{array}$ & $\begin{array}{l}\text { Average } \\
\text { life [yr] }\end{array}$ \\
\hline \multirow[t]{2}{*}{ [7] } & \multirow{2}{*}{$\begin{array}{l}\text { Squamous cell cancer of breast } \\
\text { cancer patient }\end{array}$} & \multirow[t]{2}{*}{ Overall survival rate } & With radiotherapy (WRT) & $\mathrm{I}$ & 0.050 & 20.0 \\
\hline & & & Without RT (NRT) & I & 0.086 & 11.7 \\
\hline \multirow[t]{2}{*}{ [7] } & \multirow{2}{*}{$\begin{array}{l}\text { Stage II Squamous cell cancer } \\
\text { of breast cancer patient }\end{array}$} & \multirow[t]{2}{*}{ Overall survival rate } & WRT & I & 0.040 & 25 \\
\hline & & & NRT & I & 0.120 & 8.3 \\
\hline \multirow[t]{2}{*}[16]{} & \multirow[t]{2}{*}{ Occult breast cancer (OBC) } & \multirow[t]{2}{*}{ Overall survival rate } & $\begin{array}{l}\text { After axillary lymph node } \\
\text { dissection (ALND) }\end{array}$ & I & 0.092 & 10.8 \\
\hline & & & ALND + RT & I & 0.046 & 21.7 \\
\hline \multirow[t]{2}{*}{ [17] } & \multirow[t]{2}{*}{ Renal disease dialysis patients } & \multirow[t]{2}{*}{ Overall survival rate } & Male patients & I & 0.167 & 6.0 \\
\hline & & & Female patients & I & 0.067 & 15.0 \\
\hline \multirow[t]{2}{*}{ [17] } & \multirow[t]{2}{*}{ Renal disease dialysis patients } & \multirow[t]{2}{*}{ Overall survival rate } & $\begin{array}{l}\text { Male patients with liver } \\
\text { cancer }\end{array}$ & I & 0.300 & 3.3 \\
\hline & & & $\begin{array}{l}\text { Female patients with liver } \\
\text { cancer }\end{array}$ & I & 0.400 & 2.5 \\
\hline
\end{tabular}

\section{colon cancer stage IV breakdown by patients that underwent surgical operation or not}

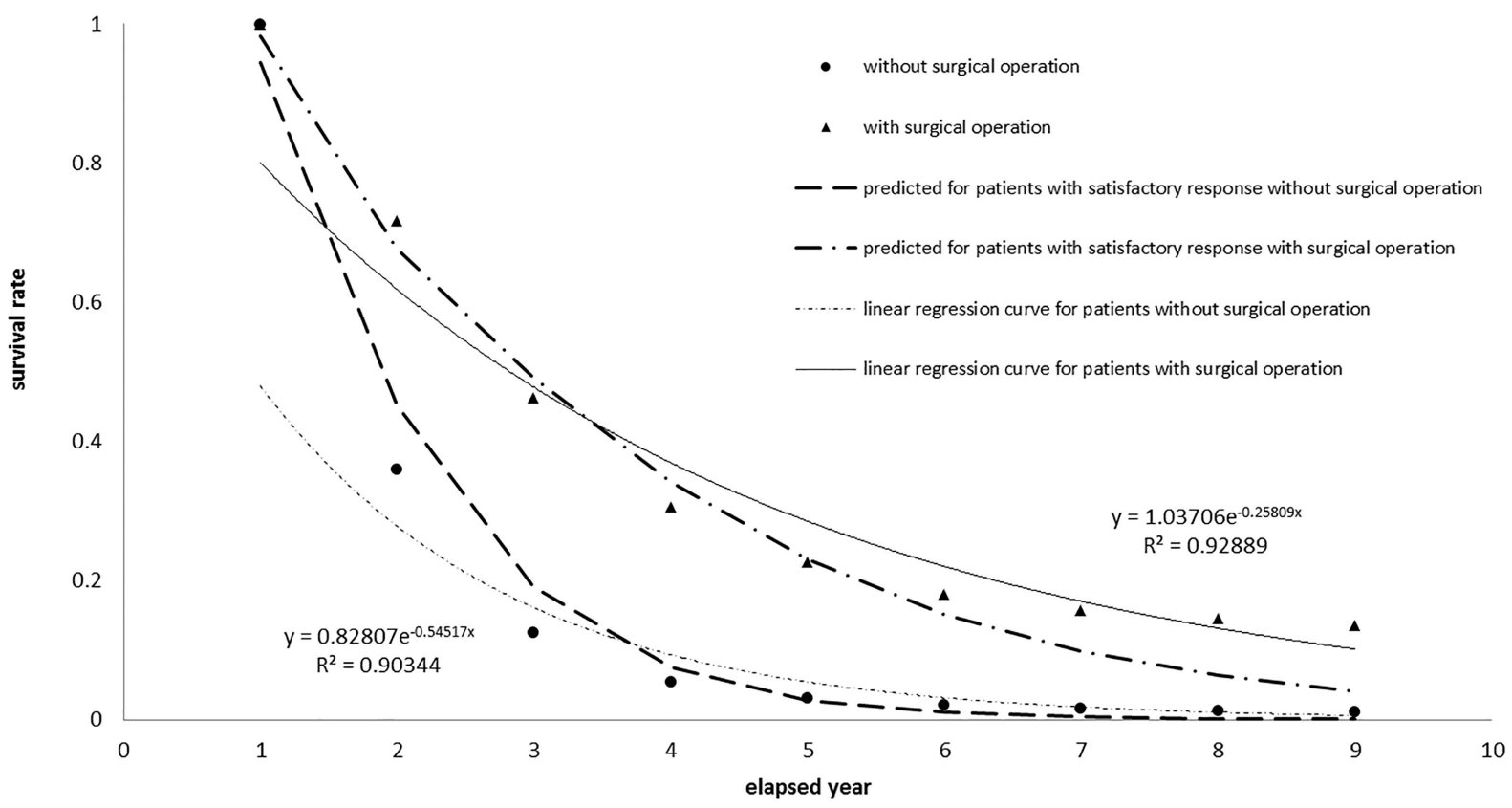

Fig. 5. Application of the proposed algorithm to colon cancer patients at stage IV recorded in Taiwan. The interpretation fitted well and implied Scenario I ("patient has satisfactory response to therapy").

response to therapy, thus, the reported data fit Scenario I "patient expired because of disease progression". As shown in Table 5 bottom row, the renal disease dialysis female patients with liver cancer had the shortest average life (2.5 year), whereas the renal disease dialysis female patients without cancer still had 15 years of average residual life. The advantage of revised algorithm is to provide a quantitative 
index, either lethal frequency or average life, in order to imply the whole survival rate curve under any specific circumstance if and only if the estimated exponential function can properly fit the original data. Therefore, the revised algorithm based on the Taylor series expansion provided the accurate prediction of (i) the survival rate of breast cancer patients, (ii) effectiveness verification of radiotherapy for colon and rectum cancer patients [7,16], and (iii) re-evaluation of the disease progression for patients of both genders reported by other researchers [17].

\section{Conclusion}

A revised algorithm to predict the survival rate of breast cancer patients at various (0-IV) stages was proposed according to Taylor series expansion and population-based survey in Taiwan (2007-2015). The algorithm can improve the accuracy in predicting the survival rate by incorporating an additional term of patient's recovery from therapy into the original survival rate regression. Four kinds of recovery for breast cancer patients undergone radiotherapy or not (WRT or NRT) were assigned, in compliance with the hit-Target model, which was earlier proposed for analyzing the cell death from the microscopic viewpoint. Accordingly, the expression of patient's survival rate can be expressed by a simple exponential function or that multiplied by an additional term to represent the recovery mechanism. The patient's recovery from the specific therapy could be assessed for different levels of shoulder effect on the survival rate prediction. A wide shoulder always implied a satisfactory response to the therapy and thus increased the survival rate. The survival rate could be quantitatively defined by the lethal frequency, which large values implied a low survival rate and short average life of the patient under study. The interpretation of lethal frequency was similar to the decay constant in the radionuclide decay process. The algorithm was also applied to interpret the survival rate of colon and rectum cancer patients at Stage IV and also exhibited a good agreement with actual collected data.

\section{Acknowledgments}

The authors would like to thank the Ministry of Science and Technology of the Republic of China for the financial support of this research (contract no. MOST 106-2221-E-166-002).

\section{Conflict of interest}

None to report.

\section{References}

[1] Cancer Registry Annual Report, Health Promotion Administration, Ministry of Health and Welfare, The Executive Yuan, Taiwan 1995-2015.

[2] Peairs KS, Barone BB, Snyder CF, et al. Diabetes mellitus and breast cancer outcomes: A Systematic review and metaanalysis. Journal of Clinical Oncology. 2011; 29(1): 40-46.

[3] Berry DA, Cronin KA, Plevritis SK, et al. Effect of screening and adjuvant therapy on mortality: Breast cancer. N Engl J Med. 2005; 353: 1784-1792.

[4] Liu FC, Lin HT, Kuo CF, et al. Epidemiology and survival outcome of breast cancer in a nationwide study. Oncotarget. 2017; 8(10): 16939-16950. 
[5] Huang PI, Yen SH. Radiotherapy for breast cancer. J. Chinese Oncol. 2008; 24(2): 121-126.

[6] Chuang WK, Cheng CT, Tsai YC, et al. Post-mastectomy radiotherapy in N1 breast cancer patients: a propensity score matching analysis using Taiwan cancer registry database. Therapeut Radiol Oncol. 2016; 23(4): 249-257.

[7] Wu SG, Sun JY, Liu WM, et al. Effect of postoperative radiotherapy for squamous cell cancer of the breast in a surveillance epidemiology and end results of population-based study. Oncotarget. 2016; 7(9): 10684-10693.

[8] Wang TH, Kittipayak S, Lin YT, et al. Quantification the in vitro radiosensitivity of Mung bean sprout elongation to 6MV X-ray: A revised target model study. Plos One. 2015; 10(6): e0128384, DOI: 10.1371/journal.pone.0128384.

[9] Turner JE. Atoms, Radiation, and Radiation Protection, 2nd ed. John Wiley \& Sons, Inc. 2000. ISBN 978-3-527-40606-7.

[10] ICD-O-3, International Classification of Diseases for Oncology, Third Edition, World Health Organization International, 2000.

[11] Cancer Staging Manual, American Joint Committee on Cancer, $8^{\text {th }}$ Ed. Springer Nature, USA, ISBN 978-3-319-40617-6.

[12] SAS, Statistical Analysis System Institute, July, 2013. https://www.sas.com/en_us/software/sas9.html.

[13] Rich JT, Neely JG, Paniello RC, et al. A practical guide to understanding Kaplan-Meier curves. Otolaryngol Head Neck Surg. 2010; 143(3): 331-336.

[14] Kuo HN, Yi JE, Chen CH, et al. Taguchi dynamic analysis application to computer tomography number-mass density linear dependence optimization, Computer Assisted Surgery, 2017. http://dx.doi.org/10.1080/24699322.2017.1378791.

[15] Yeh DM, Wang THA, Pan LK. Evaluating the quality characteristics of TLD-100T and TLD-100H exposed to diagnostic X-rays and 64 multislice CT using Taguchi's quality loss function. Radiation Measurement. 2015; 80: 17-22.

[16] Wu SG, Zhang WW, Sun JY, et al. Comparable survival between additional radiotherapy and local surgery in occult breast cancer after axillary lymph node dissection: a population-based analysis. J. of Cancer. 2017; 8(18): 3849-3855.

[17] Chien CC, Han MM, Chiu YH, et al. Epidemiology of cancer in end-stage renal disease dialysis patients: a national cohort study in Taiwan. J. of Cancer. 2017; 8(1): 9-18. 